\title{
FOSeL: Filtering by helping an Overlay Security Layer to Mitigate DoS Attacks*
}

\author{
Hakem Beitollahi \\ Hakem.Beitollahi@esat.kuleuven.be \\ Geert Deconinck \\ Geert.Deconinck@esat.kuleuven.be \\ Katholieke Universiteit Leuven \\ Electrical Engineering \\ Kasteelpark Arenberg 10, Leuven, Belgium
}

\begin{abstract}
Denial of service (DoS) attacks are major threat against availability in the Internet. A large number of countermeasure techniques try to detect attack and then filter out DoS attack packets. Unfortunately these techniques that filter DoS traffic by looking at known attack patterns or statistical anomalies in the traffic patterns can be defeated by changing the attack patterns and masking the anomalies that are sought by the filter. Hence, detecting DoS traffic is one of the main challenges for filtering techniques. Furthermore techniques that drop any malicious packet need to process the packet and processing is time-consuming.

This paper addresses how an efficient and good filter can be designed by helping an overlay network layer to mitigate DoS attacks. Fosel (Filtering by helping an Overlay Security Layer) filter is independent from DoS attack types, so we do not worry about the changing attack patterns. Furthermore it reduces processing time noticeably.

Through simulation this paper shows by employing Fosel filter, DoS attacks have a negligible chance to saturate the target by malicious packets. Our simulation demonstrates that Fosel architecture reduces the probability of successful attack to minuscule levels. Furthermore Fosel is between $10 \%$ and 50\% faster than SOS (Secure Overlay Services) [8] architecture to drop malicious packets based on attack rate.
\end{abstract}

\section{Introduction}

Today Internet is becoming an emerging technology for remote control of industrial applications, where one site needs to control another site remotely (e.g. power plants controllers) [1]. Denial-of-Service (DoS) attacks

\footnotetext{
*Acknowledgements: This work has been partially supported by the K.U.Leuven Research Council (project GOA/2007/09) and by the European Commission (project IST-4-27513 CRUTIAL)
}

may cause significant disruptions to the Internet which will threaten the operation of such network based control systems. This is because they may result in massive service disruptions and also because they have proven to be difficult to defend against.

Solving the network DoS problem is hard, given the fundamentally open nature of the Internet and the apparent reluctance of router vendors and network operators to deploy and operate new, potentially complex mechanisms. However, there are various approaches to solving DoS problems (see related work). Filtering techniques are one of the main countermeasure techniques against this problem $[4,9,11]$. These techniques try to detect attack and then drop malicious packets while these approaches may be appropriate for several types of DoS attacks; they unfortunately are not always applicable or practical. By the way every day new techniques are invented for DoS attacks that cause DoS detecting techniques become hard and harder. However, filtering techniques suffer from the following problems:

1. The accuracy with which legitimate traffic can be distinguished from the DoS traffic.

2. The methods that filter traffic by known patterns or statistical anomalies in traffic patterns can be defeated by changing the attack pattern and masking the anomalies that are sought by the filter.

3. If attackers obtain an account of one legitimate users, they can use legitimate source IP address to attack the system.

4. Filtering techniques need to process any packet to drop or accept. Unfortunately processing is timeconsuming. This problem itself can reduce availability and performance of the system.

This paper addresses how an efficient and good filter can be designed by helping an overlay network layer to mitigate DoS attacks. Fosel (Filtering by helping an Overlay 
Security Layer) is independent from DoS attack patterns. So we are not worry about changing attack patterns or accuracy detection of malicious traffic (solving problems 1, and 2). Fosel is a novel architecture that proactively protects application sites from DoS attacks. In a nutshell, the target is protected by a filter (Fosel filter) that drops any packet whose source addresses are not approved. The small set of source addresses that are approved at any particular time is kept secret so that attackers cannot use them to pass through the filter (solving problem 3). These secret nodes are selected by the application sites among the overlay network nodes. The overlay network is used to tunnel securely the legitimate traffic through several hops to the approved (secret from attackers) nodes which can then deliver legitimate traffic to the target through filter. In case of DoS attack against the target, rather than processing all arriving packets, the target's filter (Fosel filter) processes only a subset of received packets and drops all remaining packets without any processing. To ensure that legitimate messages are not lost the secret nodes deliver multiple copies of the messages to the target. Processing only a subset of arriving packets reduces processing time while legitimate packets are not lost (solving problem 4).

In this paper, we discuss how to design an overlay layer such that it is secure enough, given attackers who have a large but finite set of resources to perform the attacks. The attackers know the IP addresses of the nodes that participate in the overlay and also IP addresses of the application sites. However, a few nodes are secret for attackers.

We evaluate the Fosel architecture by simulation. There are two possibilities for attack the system: a) attack against an application site and b) attack against the overlay network. Through simulation we show that by employing Fosel filter, attackers have a negligible chance to saturate the application sites by malicious packets. For attacking the overlay, we evaluate the likelihood that an attacker is able to prevent communication among application sites. Results show that even if attackers are able to launch massive attacks (Distributed DoS) against the overlay they are unlikely to prevent successful communication. For example, in a static attack case (focused attack on a fixed set of nodes), DoS attacks are countered completely. In a dynamic attack case, if attackers can launch attacks upon $50 \%$ of nodes in the overlay, still $90 \%$ of communications among application sites are successful.

By the way we show Fosel architecture is between $10 \%$ and 50\% faster than SOS (Secure Overlay Services) [8] architecture based on attack rate. We also show Fosel architecture is a good choice for application sites that need real-time communication.

\section{Related Work}

Existing approaches generally fall into two categories: reactive techniques and proactive techniques. Former approaches need to differentiae between legitimate and malicious packets and then drop malicious packets; while the latter category does not need detection mechanisms. They prevent targets from DoS problem either by considering some controls in the network, or transport layer (e.g., rate control techniques) or by using an interface layer (e.g., hide location techniques).

Filtering techniques are reactive approaches that detect malicious packets and drop them $[4,9,11]$. For example the D-WARD defense system [10] is deployed at sourceend networks, and autonomously detects and stops attacks originating from these networks. Attacks are detected by the constant monitoring of two-way traffic flows between the network and the rest of the Internet to detect discrepancies from normal flow models and rate-limit mismatching flows in proportion to their aggressiveness.

Trace-back techniques try to find source of attack [13, 14]. The most complex issue with these approaches is that attackers often use spoofed IP addresses, hence finding real location of attackers is hard.

Rate-control techniques are countermeasures that are based on rate control and seek to enforce fairness in the bandwidth allocation, thus minimize the damages caused by DoS attacks [5, 19]. For example in Pushback [6], rate control is defined on the granularity of aggregate, which is a subset of packet flows with identifiable specific property. Pushback routers can effectively regulate any network aggregate if the corresponding property is associated with an attack. Intuitively, the attacking aggregates can be identified by the victim and then pushed back to upstream pushback routers.

Location-hiding techniques try to hide location of application sites and thereby prevent DoS attacks, which depend on the knowledge of their locations $[8,15,16,18]$. Normally overlay networks are used to hide the location of an application site. SOS (Secure Overlay Services) [8] is an architecture that uses the overlay network for hiding locations. SOS uses filters combined by secret servlets to enforce all application access being mediates through the SOS network. In the SOS architecture, access requests will be authenticated by SOAP nodes and then routed via the Chord overlay network [17] to one of the beacon nodes and then to one of the servlets, which then forwards the requests to the target site which is protected via filters. However, tolerating large scale DoS attacks (DDoS attacks) still is a challenge in the SOS architecture. 


\section{Fosel Architecture Description}

Fosel architecture is a proactive approach to prevent application sites from DoS attacks. The goal of the Fosel architecture is to provide a well-filter that can be installed on destination's routers to protect application sites from DoS attacks. Fosel allows communication only among confirmed application sites. It means that application sites have given each other a prior permission. Typically, this means that any packet must be authenticated through the Fosel architecture before the packet is allowed to forward to the destination. Fosel architecture can be used for a set of distinct nodes that communicate together by the Internet. For example the offices of a company that have been distributed in the large area (e.g., Europe or North America). Hence Fosel architecture is not suitable for a global system like Google or Yahoo.

Fosel architecture employs an overlay network that is composed of nodes that communicate atop the underlying network. The IP addresses of all overlay nodes and application sites are known and clear to the public and also to the attackers. However a certain roles that a set of nodes may be have are kept secret from public and attackers. We call these secret nodes, green nodes.

Attackers can launch DoS attacks against application sites and overlay nodes from variety points of the Internet. As any application site connects to the overlay by the authentication technique, we assume that attackers cannot penetrate inside the overlay and so they cannot send malicious packets inside the overlay. Figure 1 shows a highlevel overview of the Fosel architecture that protects application sites from DoS attacks. In the following subsections the architecture is explained step by step.

\subsection{Architecture and Operation Design Rationale}

The goal of Fosel architecture is to provide a immune sub-network from DoS attacks on the Internet among the application sites of a company. Any application site connects a random overlay node to send a data, a request or a message for any other application site. The overlay node analyses and verifies the message by authentication techniques. If the message is authorized successfully it is allowed entering inside the overlay, otherwise it is dropped by the overlay node. Hence, at a very basic level, we need the functionality of firewalls in the overlay network that use authentication techniques to drop malicious packets. Authentication techniques can be solved by using traditional protocols such as IPsec, TLS, or by smart cards. Thereby attackers cannot penetrate inside the overlay.

After a legitimate message was verified by the overlay node it is forwarded and routed through the overlay nodes

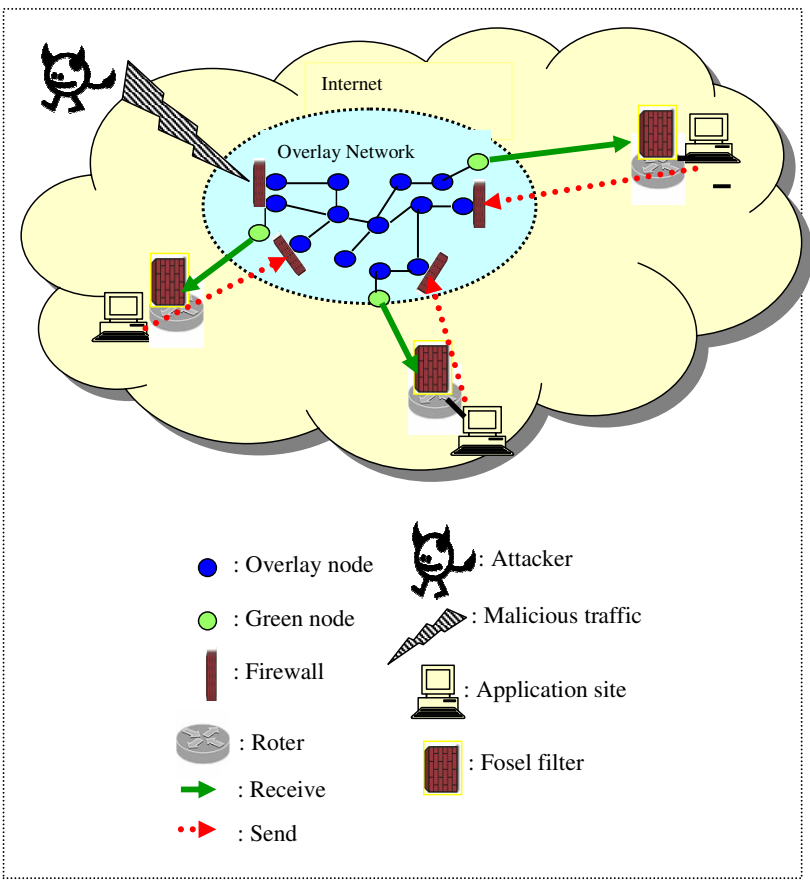

Figure 1. The Fosel architecture

to the secret green node of the application site. Then green node delivers the message to the application site (final destination) through the Fosel filter.

In case of DoS attack against an application site the green node of the application site delivers multiple copies of the message to the application site, otherwise (no DoS attack) delivers one copy of the message to the application site.

\subsection{The application site filter (Fosel filter)}

The IP address of an application site is the sufficient information for attackers to attack the application site by DoS attack. To prevent such attacks a Fosel filter can be constructed and installed on destination's routers. So malicious and illegitimate packets are dropped by the Fosel filter. We assume attackers do not have access inside the destination's routers and filter region. Past history indicates that it is significantly more difficult for an attacker to take access of a router and poison the filter (compared to, e.g., a web server or a desktop computer) [8]. Hence, we assume that attackers cannot penetrate inside the router.

We assume the Fosel filter is installed on high-powered final routers such that i) these routers can deliver high load of data to the destination and ii) possibly there are several disjoint paths leading the application site (an application site has some routers on disjoint paths). So if one of these paths is brought down, the message can traverse from other paths to the application site. 
Fosel filter (final filter) accepts only those packets whose come from the green nodes of the application site. Hence Fosel filter drops all other packets whose their source address does not match the address of its green nodes. Fosel filter does not need to verify signature and decrypt data. It just need to see IP address of a message. If it (IP address) belongs to its green nodes, the messae is accepted otherwise it is dropped. This simple and straightforward approach has two main advantages: i) when an application site changes its location and moves to another location (change IP) the filter does not need to be notified and then modified, because it is independent from users location (the application sites IP). ii) Attackers cannot use spoofed IPs to attack the target. As IP of application sites is clear to the public, attackers can use their IPs to attack the target, but as Fosel filter only accepts data from secret green nodes, attackers cannot attack the target by spoofed and bogus IPs.

Because of the small number of such filter rules and simple nature (filter only on source IP address), the time for processing a packet is decreased to at least $2 / 3$ of time required by other filters (filter on signature and data decryption). Suppose any read, decrypt and compare operation takes one T. Other filters need 3T (read, decryption and compare) to process any packet while Fosel filter need 2T (read and compare).

Second task of Fosel filter is that in case of DoS attack rather than process all arriving packets, process only a subset of the received packets. To ensure that legitimate messages are not lost the green nodes of the application site transmits multiple copies of each message. Upon fist observation, this technique appears to worsen the DoS attack by increasing the amount of traffic received by the application site (multiple copies of each message). However, due to asymmetry in the cost of sending versus processing messages, the large reduction in processing cost outweighs the increase in network traffic during an attack. For the green nodes, sending multiple copies of message is cheap. To alleviate this potential high cost (increase traffic by multiple copies), Fosel filter randomly drops any packet with probability of P. By this approach most of bogus packets are discarded and dropped without any processing while due to multiple copies of a message, the legitimate message has a high chance to be processed. Through simulation or analytical analysis for any value of $\mathrm{P}$ and under any attack rate, the number of message copies can be determined with this condition that the miss rate is less than $\sigma$ (e.g., $\sigma=0.0001$ ).

\subsection{Green nodes}

In a short sentence green nodes are secret nodes of the overlay network. The role of green nodes is kept secret and hence nobody knows which nodes are green nodes. The overlay network, along with the green nodes helps the ap- plication site to have effective and fast filter (Fosel filter) to drop malicious packets.

To activate a green node, the application site sends a message to the overlay node that it chooses to be a secret green node, informing it of its task. Any application site may have a few green nodes. Fosel filter in the destination is set to only pass packets whose source address matches the address of the green nodes of the destination.

A green node, upon receiving a packet from the overlay, forwards it to the destination through Fosel filter. In case of DoS attack it delivers multiple copies of the message.

A question may be made that attackers can guess the routing path and then disclose the location of green nodes. The answer is no, because overlay networks such as Chord, or CAN [12] have the complicated routing mechanisms that will route packets to destination efficiently, while utilizing a minimal amount of information about the identity of that destination. Overlay networks have a dynamic nature and high level of connectivity. In these networks unlike the underlying network an edge is permissible between any pair of overlay nodes, hence overlay networks have flexibility and several choices to select a route that complicates the job of attackers to determine the path taken within the overlay to a secret green node.

\subsection{Attacking the Overlay}

Attackers can attack the overlay from variety points of the Internet simultaneously. However, these attacks have no influences on application sites. Overlay network can tolerate these attacks due to their dynamic nature and high level of connectivity. Since a path exists between every pair of nodes, it is easy to recover from a breach in communication that is the result of an attack that shuts down a subset of overlay nodes. The recovery action simply removes those "shut down" nodes and then the overlay reconfigures itself (update hashing and routing tables). Furthermore, no overlay node is more important or sensitive than others.

If a green node identity is disclosed and the green node is targeted as an attack point, that green node is simply removed from the overlay and the target (the application site) chooses another node randomly as a new green node.

\subsection{Some Additional Points}

- The Fosel architecture utilizes the Chord network as an overlay network [16]. Chord network has some valuable properties that encourages the using the Chord network in the Fosel architecture.

- In a Chord network, to find a key X from any node, $\mathrm{O}(\log \mathrm{N})$ steps are required. In fact a Chord node only needs a small amount of routing information about other nodes. 
- Chord network never partitions. It means that if attackers attack the Chord network simultaneously and bring down many nodes, Chord easily reconfigures itself without partitioning. In fact Chord is able to route effectively even if only one node remains in the overlay.

- Chord network is the most dependable network compared to other overlay networks $[7,2]$.

- An application may have some green nodes (redundancy in green nodes) due to fault tolerance policy.

- An application site can understand that it is under DoS attack just by checking the threshold of arriving packets. If arriving rate is more than $\beta$ (threshold), it is under DoS attack and sends a message to its green nodes that I am under attack. Now green nodes send multiple copies of a message instead of one copy.

- The receiver may process multiple copies of a legitimate message. Nonce can be used to quickly discard duplicates. Nonce can be analyzed in destination after acceptance of the message by Fosel filter (the message has passed the filter).

\subsection{Summary of Architecture}

To summarize the sequence of operation in the Fosel architecture, we explain sending operation both in case of attack-free (no DoS attack) and in case of DoS attack.

- Sending operation in case of no DoS attack (attackfree):

1. An application site sends its message to a random overlay node.

2. The overlay node verifies the message by authentication technique. If it is ok the message is accepted; otherwise it is dropped.

3. The overlay node sends the message to the green nodes of the destination.

4. The green nodes deliver message to the destination through Fosel filter.

5. Fosel filter checks source address of the message: if it is from green nodes it is accepted; otherwise it is dropped.

- Sending operation in case of DoS attack against application site "A":

1. The application site "A" (receiver) sends a message to its green nodes that it is under DoS attack.

2. An application site (sender) sends its message to a random overlay node.
3. The overlay node verifies message by authentication technique. If it is ok the message is accepted; otherwise it is dropped.

4. The overlay node sends the message to the green nodes of the application site "A".

5. The green nodes deliver $\mathbf{C}$ copies of message to the application site through Fosel filter.

6. Fosel filter drops any message (malicious or legitimate) with probability of $P$ without any processing and process the remaining packets.

7. Fosel filter checks source address of the remaining messages: if the message is from the green nodes it is accepted; otherwise it is dropped.

\section{Performance evaluation of Fosel architec- ture}

In this section we analyze the performance of the Fosel architecture by simulation. First some points:

- Attackers know IP of the set of overlay nodes and also application sites. So attackers can start DoS attacks against them.

- Attackers have a bounded and fixed amount of bandwidth to attack the architecture. For instance, attackers can attack maximum $\mathrm{X}$ nodes $(X<N, \mathrm{~N}$ is the total number of overlay nodes) simultaneously.

- Attackers do not know which nodes are green nodes.

- Attackers cannot penetrate inside the overlay due to strong authentication techniques and firewalls.

- Each application site can access the overlay through access control techniques (via firewalls).

- Each application site has 5 green nodes.

- 20 senders simultaneously send messages to an application site.

\subsection{Implementation}

We implemented a simulator to evaluate the Fosel architecture. To do this we implement the Chord protocol (main backbone of Fosel architecture), attack toolkit, and Fosel filter (destinations' filter).

- Implementing the Chord network: We have implemented the Chord network in an iterative style [17]. In the iterative style, a node resolving a lookup initiates all communications: it asks a series of nodes for 
information from their hashing tables, each time moving closer on the Chord ring to the desired successor. During each stabilization protocol step, a node updates its immediate successor and one other entry in its successor list or hashing table. Thus if a node's successor list and hashing table contain a total of $\mathrm{k}$ unique entries, each entry is refreshed once every $\mathrm{k}$ stabilization rounds. When the predecessor of node $\mathrm{m}$ changes, $\mathrm{m}$ notifies its old predecessor $q$ about the new predecessor q'. This allows q to set its successor to q' without waiting for the next stabilization round.

The delay of each packet is exponentially distributed with an average of $50 \mathrm{~ms}$. If a node $\mathrm{m}$ cannot contact another node m' within $500 \mathrm{~ms}$, m concludes that $\mathrm{m}$ ' has left or has been attacked (we consider same action for both leaving node and attacked node although we suppose different rates for these). If m' is an entry in m's successor list or hashing table, this entry is removed. Otherwise $m$ informs the node from which it learns that $\mathrm{m}^{\prime}$ is gone.

- Implementing the attack toolkit: Attackers are placed outside the overlay. To implement an attack toolkit, we program basic structure of Trinoo [3] to generate both DoS and DDoS attacks by $\mathrm{C}++$. In fact we implement two basic procedures for attack toolkit: daemon and master procedures. We have several daemon procedures that are controlled by master procedure. Daemon procedures simply send malicious traffic to the targets at the given start time that determined by master procedure. We consider both static and dynamic attacks. In a static attack, attackers select a fixed set of overlay nodes to attack and when an attacked node is removed from the overlay, the attacker cannot redirect to another node. In the dynamic approach, attackers can attack any node and also can redirect attacks to other nodes.

- Implement Fosel filter (receiver's filter):Fosel filter utilizes q queues (total queues). Incoming messages (legitimate or malicious) are enqueued into queues at random. Then filter selects $\mathrm{k}$ queues at random for processing and drops q-k queues without any processing. For processing a message, filter only checks the source address of the message. If source address is an address of one of its green nodes, the message is accepted otherwise it is dropped. In the rest of the paper receiver refer to the Fosel filter (receiver's filter).

\subsection{Simulation results}

There are two possibilities to attack the system: attacking the application sites and attacking the overlay network.

\subsubsection{Attacking the application sites}

A sender's message maybe miss to be processed due to random selection of $\mathrm{k}$ queues from q queues (total queues) for process. It is therefore possible that due to miss probability, no copies of a particular message are present in any of the selected queues. We call this a miss and the fraction of unique sender requests that end in miss the miss rate. So miss rate is: number of unprocessed senders per total number of senders.

miss_rate $=$ unprocessed_senders/total_number_of_senders.

Here, we show that by checking only a small number of queues, the miss rate becomes quite low. We can suppose two states for an application site: a) unlimited length for queues and $b$ ) limited length for queues. We suppose that receiver has totally 20 queues $(\mathrm{q}=20)$.

\section{queues with unlimited length:}

Figure 2 shows miss rate for different values of selected queues (different values of $\mathrm{P}, p=k / q$ ) when the number of message's copies is varies along the $\mathrm{X}$-axis. $\mathrm{K}$ is the number of selected queues.

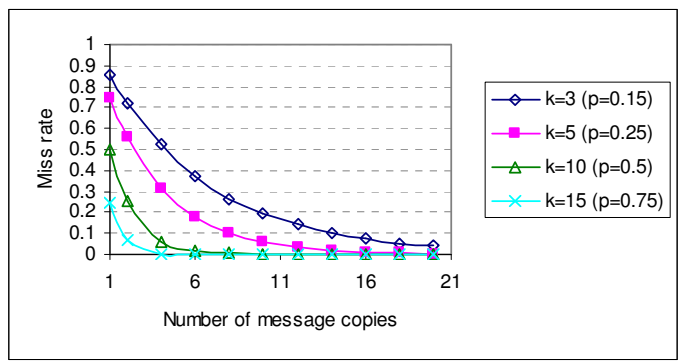

Figure 2. Miss rate vs. number of message copies (20 senders simultaneously)

In the first observation, simulation results show by increasing number of message copies the miss rate is decreased. Simulation results show that by selecting 10 queues from totally 20 queues $(\mathrm{P}=0.5)$, a green node needs to send just 5 copies of a message (in attack time) to have miss rate less than $1 \%$.

Figure 3 shows miss rate for different values of message copies when the number of selected queues varies along $\mathrm{X}$ axis. $\mathrm{C}$ is number of message copies.

Both figures 2 and 3 show the miss rate is decreased when the number of message copies or the number of selected queues is increased. Figure 3 also again shows a green node needs to send just 5 copies of a message when 


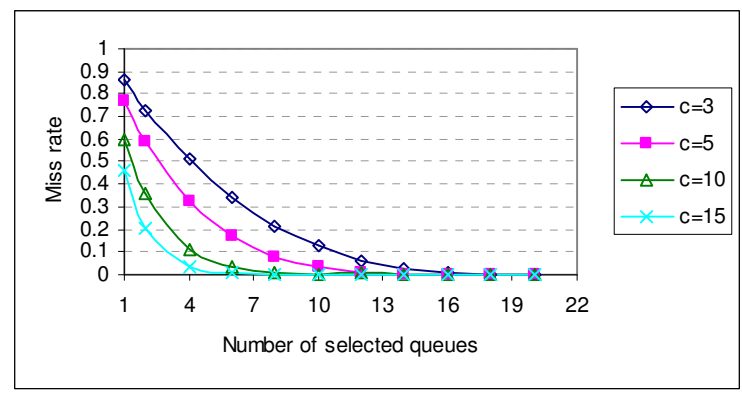

Figure 3. Miss rate vs. number of selected queues (20 senders simultaneously))

\begin{tabular}{|c|c|}
\hline Number of selected queues & Number of message copies \\
\hline $\mathrm{K}=3(\mathrm{P}=0.15)$ & $\mathrm{C}=20$ \\
\hline $\mathrm{K}=5(\mathrm{P}=0.25)$ & $\mathrm{C}=15$ \\
\hline $\mathrm{K}=10(\mathrm{P}=0.5)$ & $\mathrm{C}=5$ \\
\hline $\mathrm{K}=15(\mathrm{P}=0.75)$ & $\mathrm{C}=3$ \\
\hline
\end{tabular}

Table 1. Number of selected queues vs. number of message copies (miss rate less than $1 \%$

receiver selects 10 queues for processing $(\mathrm{P}=0.5)$ to have miss rate less than $1 \%$.

From these figures ( 2 , and 3 ), we can extract useful information for the system. Table 1 shows the number of message copies that a green node needs to send during the attack time when receiver selects different number of queues for processing (miss rate less than 1\%). This table shows our hand is open. If sending more copies of messages is cheaper than processing more selected queues, we can send more copies of messages (e.g., $c=20$ ) but instead we can process less selected queues (e.g., $\mathrm{k}=3$ ) and vice versa.

\section{Queues with limited length:}

In this section we suppose queues have limited length (reality) and we show the efficacy of our new approach against DoS attacks. When queues have unlimited length, the attack rate amount does not have affect on performance because however the message copies can be enqueued and are processed finally. However when queues have limited length, the message copies maybe are not enqueued due to large amount of attack traffic and so some messages are not processed. So the amount of attack traffic has effect on performance definitely.

Figure 4 shows miss rate for different values of selected queues when the amount of attack rate varies along the $\mathrm{x}$ axis. In this experiment we hold $\mathrm{C}$ at 10.

Simulation result (figure 4) shows when the attack rate

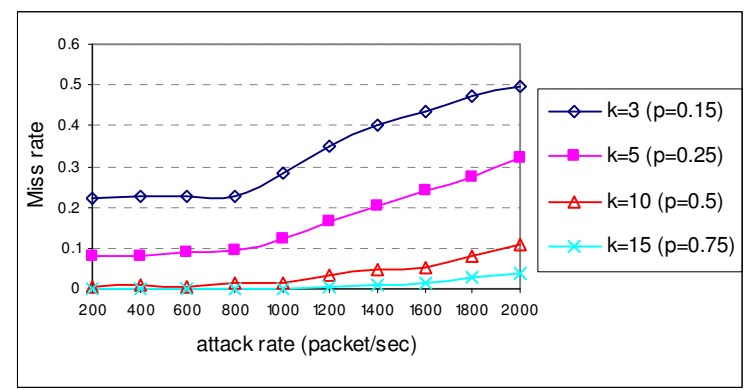

Figure 4. Miss rate vs. attack rate (for different values of selected queues

amount is increased the miss rate is increased. However, by increasing number of selected queues for processing we can reduce the miss rate. For instance by selecting 10 queues for processing the miss rate is less than $10 \%$ when the attack rate is in the maximum amount (2000 packet/sec).

Figure 5 shows miss rate for different values of message copies when the amount of attack rate varies along the $\mathrm{x}$ axis. We hold $\mathrm{k}$ at 10 in this experiment. Result shows by increasing copy numbers of a message we can thwart the affect of large attacks.

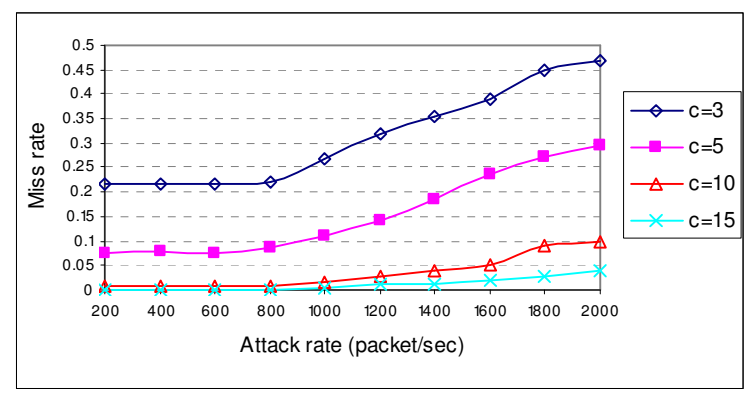

Figure 5. Miss rate vs. attack rate (for different values of message copies

Another interesting view of these figures ( 4 and 5) is that the miss rate does not increase or increase by low rate (low dip) when the attack rate starts to increase. For instance figure 5 shows for $\mathrm{C}=10$ or 15 (number of message copies) the miss rate is less than $1 \%$ when attack rate is less than 1000 packet/sec. By reducing number of message copies or number of selected queues, this amount (attack rate) should be less to have no change in the miss rate. For example when $\mathrm{C}=3$, the attack rate should be less than 800 packet/sec to have small change in the miss rate. Honestly we have no reason for such behavior of the system at this moment. However, as a result of these experiments (figures 4 and 5) we can summarize that for any value of attack rate 
the best values can be found for number of selected queues and number of message copies by simulation or analytical model in the real system.

\subsubsection{Comparing Fosel approach with SOS and straightforward filter}

In this section we compare our new approach with SOS architecture and straightforward filter (simple filter). We use time metric for comparison. In fact the time required for processing messages regardless of whether the message is legitimate, a duplicate, or malicious is compared.

Let the time require for any action be unit time T. For example any action like read, decryption or comparison is done in a T. straightforward filter needs 3T for process any packet (read, decryption, and comparison). SOS architecture needs 2T (read and comparison) and finally Fosel architecture needs 2T (read and comparison). Note that SOS and Fosel do not need decryption due to checking only source address of a message.

Let $\alpha$ be the attack rate (i.e., the number of attack messages arriving at the receiver per second), $q$ be the number of queues at receiver and $s$ be the number of senders. When straightforward filter is used, the time require for process all arriving messages is $T_{\text {straightforward }}=3(\alpha+s) T$. For SOS architecture this time is $T_{S O S}=2(\alpha+s) T$. When Fosel architecture is used this time is $T_{\text {Fosel }}=2 k / q(\alpha+$ $m s) T$, Where $m$ is the number of message copies and $k$ is the number of selected queues.

Thus, when attack rate $\alpha$ is much larger than $s$ and $m$, then the attack rate is diminished by a factor of $k / q$. For instance when $\mathrm{k}=10$ (miss rate less than $1 \%$ ) we would expect to process only $1 / 2$ of the DoS packets. Our simulation confirms our analysis. Figure 6 shows the time require for process all packets for Fosel, SOS, and straightforward filter. 20 senders send messages simultaneously.

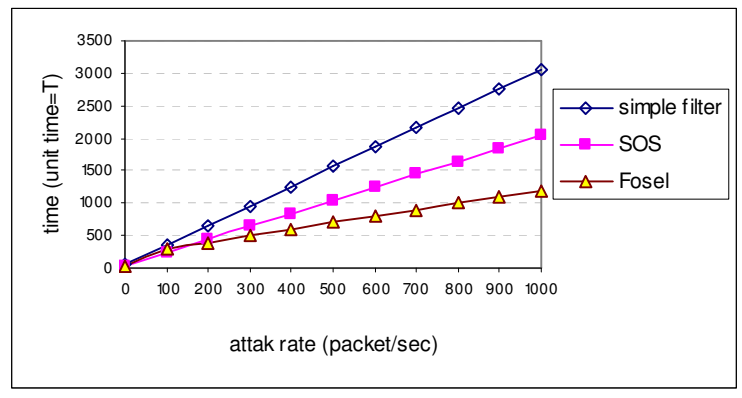

Figure 6. Compare Fosel, SOS and straightforward (simple) filter (time required for process all packets)

\section{Messages with deadline}

In this section we compare Fosel, SOS and straightforward filter when a message should be processed before its deadline. We assign deadlines to the messages based on uniform distribution between $100 T \leq$ deadline $\leq 500 T$. We suppose queues have limited length (maximum 50 messages). Figures 7 and 8 show deadline miss rate for all Fosel, SOS and straightforward filter when number of selected queues and number of message copies varies along the $\mathrm{x}$-axis respectively. Here a deadline miss message is that no copy of a message has been processed or processed after the message's deadline. Results show Fosel is far better than SOS and straightforward filter.

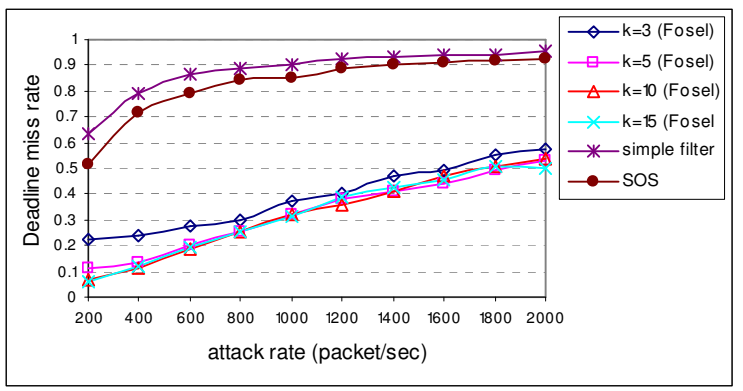

Figure 7. Compare Fosel, SOS and straightforward (simple) filter (deadline miss rate vs. attack rate for different values of selected queues)

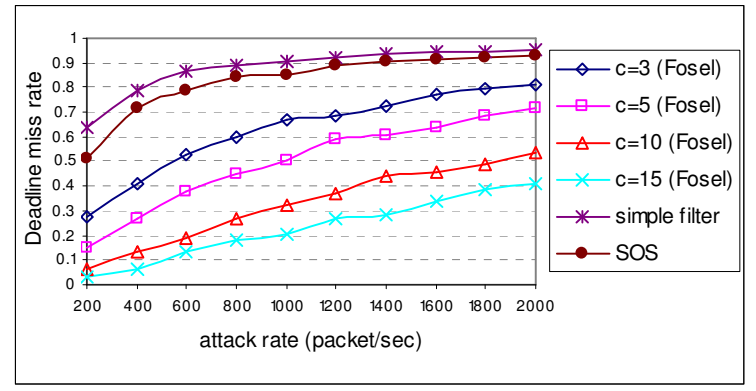

Figure 8. Compare Fosel, SOS and straightforward (simple) filter (deadline miss rate vs. attack rate for different values of message copies)

\subsubsection{Attacking the overlay network}

In this section we suppose attackers attack the overlay nodes randomly and maybe some of these attacked nodes are 
green nodes. Our evaluation in this section determines the probability of successful searches and vice versa probability of successful attack. A search is successful if a path between any two arbitrary application sites can be found.

First set of experiments analyzes the likelihood of successful searches in the static case (an attacker would select a fixed set of nodes to attack and Fosel architecture takes no action towards repairing the attack).

Figure $9(\mathrm{a}$ and $\mathrm{b})$ shows the probability of successful search and probability of successful attackers when the number of attacked nodes varies along the $\mathrm{x}$-axis, respectively.

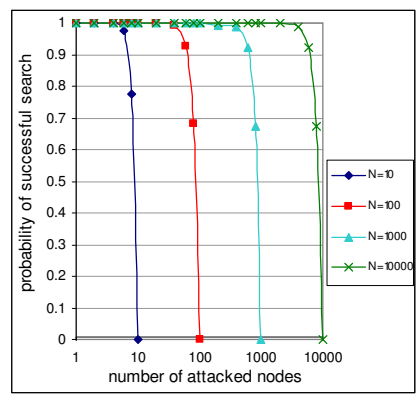

a) Probability of successful search

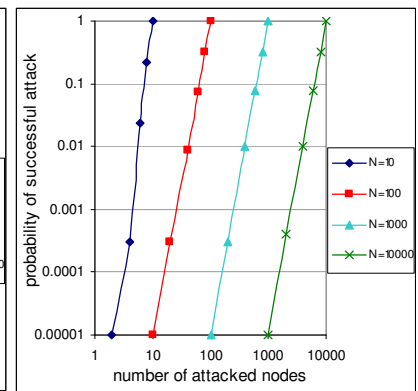

b) Probability of successful attack
Figure 9. DoS attack in static case

From this figure, we see that the likelihood of an attack successfully terminating communication between any two arbitrary application sites is negligible unless the attacker can simultaneously bring down a significant fraction of nodes in the network. For instance, if an attacker attacks $10 \%$ of overlay nodes simultaneously we have around 99.999\% successful searches. If attackers bring down between $20 \%$ and $40 \%$ of overlay nodes, more than $98 \%$ successful searches exist. Even if attackers bring down half of total nodes (50\%), there are still $90 \%$ successful searches.

Second set of experiments in this section evaluates the Fosel architecture in a dynamic case. Previous experiment assumed that an attacker would select a fixed set of nodes to attack, and that Fosel takes no action towards repairing the attack. The scenario of this set of experiments is that when Fosel architecture identifies an attacked node, that node is removed from the overlay. When an attacker identifies that a node it is attacking no longer resides in the overlay, it redirects its attack towards a node that does still reside in the overlay. When the attacked node was removed, the attack against that node terminates and the node comes back the overlay after $D_{r}$ delay. $D_{r}$ is a repair delay for reconfiguration. Also, there is an attack delay, $D_{a}$, that equals the difference in time between when an attacked node is removed from the overlay to the time when the attacker (realizing the node it is attacking has been removed) redirects the attack towards a new node in the overlay. We assume both $D_{a}$ and
$D_{r}$ are exponentially distributed random variables with respective rates $\lambda$ and $\mu$. We evaluate the Fosel architecture in the dynamic case for both DoS and DDoS attacks.

Figure $10(\mathrm{a}$ and $\mathrm{b})$ plots probability of successful searches and successful attacks respectively for DoS attack, where $\rho=\lambda / \mu$ varies along $\mathrm{x}$-axis. When $\rho \leq 1$ for any value of overlay nodes $(\mathrm{N})$, attackers are least likely ( $0 \%$ ) to deny service. Also for large value of $\mathrm{N}$ (e.g. $\mathrm{N}=1000$ ) attackers do not have chance to deny service. Even when $\rho$ is 10 and $\mathrm{N}$ is 100 , still we have about $100 \%$ successful search. We think in practice and reality always $\rho$ is less than 10. As a result DoS attack in the Fosel architecture with repair is solved nearly completely.

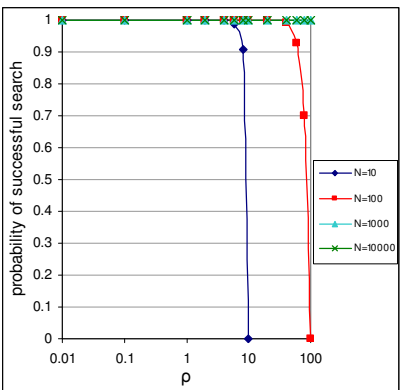

a) Probability of successful search

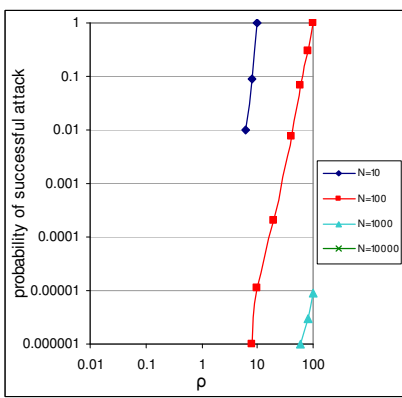

b) Probability of successful attack

\section{Figure 10. DoS attack in dynamic case}

Figure 11(and b) plots the probability of successful searches and successful attacks respectively for DDoS attack in the dynamic case. In this figure $n_{a}$ is the maximum ability of attackers that can attack simultaneously. In figure 11 simulation is done for $\mathrm{N}=1000$.

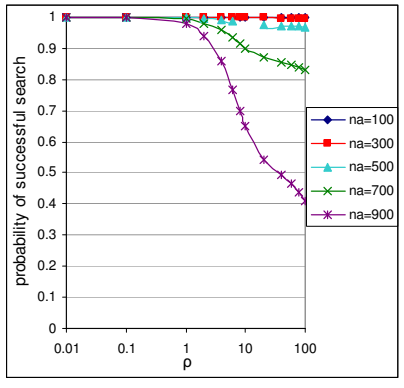

a) Probability of successful search

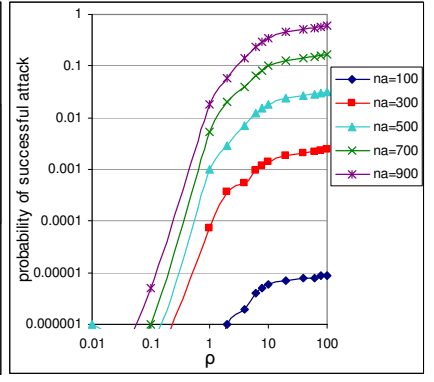

b) Probability of successful attack

\section{Figure 11. DDoS attack in dynamic case}

This figure shows that when an attack is distributed (DDoS), the fraction of time for which the attack is successful can be significant when a large fraction of nodes in the overlay is attacked. From this figure we can understand although DDoS attack is harder to tolerate than DoS attack, for $n_{a} \leq N / 2$ it can be tolerated. For instance, when $n_{a} \leq N / 2$ for any value of $\rho$ the probability of successful 
search is more than $90 \%$. An interestingly note of figure 11 is that when $\rho$ is increased above 10 , the curves reach steady state and remain constant. It means if $\rho$ is increased even more than 100 , the probability of successful attack will not change or change unnoticeably.

\section{Conclusion}

This paper addressed how a well filter can be designed by an overlay network layer to mitigate DoS attacks. Fosel architecture is a proactive technique against DoS attacks that is composed of a target filter that accepts data only from secret green nodes, an overlay network with secret green nodes that makes a secure way to deliver data to the target and firewalls with access control techniques that allow only legitimate users to use the overlay network. In case of DoS attack against an application site the filter drop any packet with probability of $\mathrm{P}$ without any process. To make sure legitimate messages are not lost multiple copies of a message is sent.

Through simulation we show that by employing Fosel filter, attackers have a negligible chance to saturate the application site by malicious packets. For attacking the overlay, we evaluate the likelihood that an attacker is able to prevent communication among application sites. Results show that even the attackers are able to launch massive attacks (DDoS) against the overlay they are very unlikely to prevent successful communication. We also show Fosel architecture is between $10 \%$ and $50 \%$ faster than SOS (Secure Overlay Services) architecture to drop malicious packets based on attack rate.

\section{References}

[1] M. Chow and Y. Tipsuwan. Network-based control systems: a tutorial. In Proceeding of the IEEE Industrial Electronics SOc., Denver, CO, pages 1593-1602, 2001.

[2] F. Depaoli and L. mariani. Dependability in peer-to-peer systems. IEEE Transaction on Internet Computing, 8(4):5461, July and August 2004.

[3] D. Dittrich. The DoS project's trinoo distributed denial of service attack toll. University of Washington, 1999.

[4] P. Ferguson and D. Senie. Network ingress filtering: defeating denial of service attacks which employ ip source address spoofing. In Proceedings of the IETF,RFC2267, January 1998.

[5] A. Garg and A. N. Reddy. Mitigation of dos attacks through qos regulation. In Proceedings of the 10th IEEE International Workshop on Quality of Service, 2002.

[6] j. Ioannidis and S. Bellovin. Implementing pushback: Router-based defense against ddos attacks. In Proceedings of the ISOC network and distributed system security symposium (NDSS), 2002.
[7] P. Karwaczynski and J. Kwiatkowski. Analysis of overlay network impact on dependability. In proceedings of the 38th Hawaii International conference on system science, 2005.

[8] A. Keromytis, V. Misra, and D. Rubenstein. Sos: Secure overlay services. In Proceedings of the Conference on Applications, Technologies, Architectures, and Protocols for Computer Communications (SIGCOMM'02), August 2002.

[9] J. Li, J. Mirkovic, M. Wang, P. Reiher, and L. Zhang. Save: Source address validity enforcement protocol. In Proceedings of the 21st Annual Joint Conference of the IEEE Computer and Communications Societies (INFOCOM'02), 2002.

[10] J. Mirkovic and P. Reither. A taxonomy of ddos attack and ddos defense mechanisms. ACM computer communication Review, 34(2), April 2004.

[11] K. Park and H. Lee. on the effectiveness of route-based packet filtering for distributed dos attack prevention in power-law internet. In Proceedings of the Conference on Applications, Technologies, Architectures, and Protocols for Computer Communications (SIGCOMM'01), pages 15-26, 2001.

[12] S. Ratnasamy, P. Francis, M. Haudley, R. Karp, and S. Shenker. A scalable content addressable network. In Proceedings of the Conference on Applications, Technologies, Architectures, and Protocols for Computer Communications (SIGCOMM’01), pages 27-31, 2001.

[13] S. Savage, D. Wetherall, A. karlin, and T. Anderson. Network support for ip traceback. ACM/IEEE Transactions on Networking, 9(3):226-237, June 2001.

[14] A. Snoeren. Hash-based ip traceback. In Proceedings of the Conference on Applications, Technologies, Architectures, and Protocols for Computer Communications (SIGCOMM'01), pages 3-14, 2001.

[15] A. Stavrou and et al. Websos: An overlay-based system for protecting web servers from denial of service attacks. the International Journal of Computer and Telecommunications Networking, 48(5):781-807, August 2005.

[16] I. Stoica, D. Adkins, S. Zhuang, S. Shenker, and S. Surana. Internet indirection infrastructure. In Proceedings of the Conference on Applications, Technologies, Architectures, and Protocols for Computer Communications (SIGCOMM'02), 2002.

[17] I. Stoica, R. Morris, D. Karger, F. Kaashoek, and H. Balakrishnan. Chord: A scalable peer-to-peer lookup service for internet applications. In Proceedings of the Conference on Applications, Technologies, Architectures, and Protocols for Computer communications (SIGCOMM'01), pages 149160, 2001.

[18] J. Wang, L. Lu, and A. Chien. Tolerating denial-of-service attacks using overlay networks-impact of topology. In Proceedings of the ACM Workshop on Survivable and SelfRegenerative Systems, 2003.

[19] K. Yau, C. Lui, and F. Liang. Defending against distributed denial of service attacks with max-min fair server-centric router throttles. In Proceedings of the IEEE International Workshop on Quality of Service (IWQoS'02), 2002. 